\title{
La recherche dans les universités du Grand Ouest
}

Thèses et HDR soutenues en 2017. Histoire - Histoire de l'Art Archéologie

\section{(2) OpenEdition}

Journals

Édition électronique

URL : https://journals.openedition.org/abpo/4317

DOI : $10.4000 /$ abpo.4317

ISBN : $978-2-7535-7720-6$

ISSN : 2108-6443

Éditeur

Presses universitaires de Rennes

\section{Édition imprimée}

Date de publication : 18 décembre 2018

Pagination : 197-200

ISBN : 978-2-7535-7718-3

ISSN : 0399-0826

Référence électronique

"La recherche dans les universités du Grand Ouest », Annales de Bretagne et des Pays de l'Ouest [En ligne], 125-4 | 2018, mis en ligne le 18 décembre 2020, consulté le 27 mars 2023. URL : http:// journals.openedition.org/abpo/4317; DOI : https://doi.org/10.4000/abpo.4317

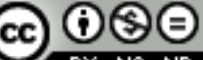

Creative Commons - Attribution - Pas d'Utilisation Commerciale - Pas de Modification 4.0 International - CC BY-NC-ND 4.0

https://creativecommons.org/licenses/by-nc-nd/4.0/ 


\title{
La recherche dans les universités du Grand Ouest
}

\author{
Thèses et HDR soutenues en 2017 \\ Histoire - Histoire de l'Art - Archéologie
}

(précédente publication en décembre 2017, ABPO 124-1)

\section{Université d'Angers}

\section{Thèses}

ARFuch, Diego Elias, Hagiographica et dogmatica cypria. Estudio sobre el cristianismo chipriota del siglo val VII en la consolidacion de la autocefalia. Expresiones canonicas, dogmaticas, liturgicas y hagiograficas. Directeur : Philippe BLAUDEAU.

GODET, Antoine, La symbolique politique des mouvements fascistes et fascisants en France et en Grande-Bretagne dans les années 1930. Étude comparative de la British Union of Fascists et du Parti populaire français. Directeur : Yves DENÉCHÈRE.

MALETH, Agnès, L'évolution de la politique occidentale de la Hongrie angevine: la papauté, la France et l'Allemagne (1301-1342). Directeurs : Jean-Michel Matz et S. Cernus (Université de Szeged).

Roul, Jehanne, Le poème en l'honneur de Louis Le Pieux d'Ernold Le Noir : un poème pour l'empire carolingien. Directeur : Thomas DESWARTE.

\section{Université de Bretagne occidentale (Brest)}

Thèse

Delumeau, Isabelle, La carte nautique et ses usages (vers 1830 - vers 1880). Directeur : Philippe JARNOUX.

MARGOUT, Thomas, Le soucoupisme français : 1945-2012. Directeur : Fabrice BOUTHILLON.

Kobela, Emmanuel Alain, L'impact des projets de développement sur la qualité de vie des femmes : l'exemple du PRODALKA au Tchad. Directrice : Arlette GAUTIER.

LE Roux, Gael, L'alcoolisation des étudiantes irlandaises. Directrice: Arlette GAUTIER.

\section{Université de Bretagne Sud (Lorient)}

\section{Thèse}

DANA, Katherine, Rennes et la mer. Création, développement et fonctionnement d'une voie fluviomaritime : la 
Vilaine (XVI ${ }^{e}$-XVII siècles). Directeur : Gérard LE BOUËDEC.

Toussaint, Frédéric, Hennebont de la lumière à l'ombre : 1660-1780. Directrice : Sylviane LLINARES.

\section{Université de La Rochelle}

\section{Habilitation à diriger des recherches}

TRANCHANT, Mathias, Les ports maritimes de la France atlantique aux $X I^{e}-X V^{e}$ siècles, $4 \mathrm{vol}$.

\section{Thèses}

MORILlon, Sébastien, Bernard Charbonneau : agir pour "sauver la nature et la liberté », des années 1930 aux années 1968, La Rochelle, 2017. Directeur : Didier PотоN.

\section{Université du Maine (Le Mans)}

\section{Thèses}

Khatchadourian, Anaïs, Mousa Sadr. Biographie intellectuelle d'un clerc chiite. Directeurs : Dominique Avon et Jean-François LEMOINE.

MARCHAL, Sidonie, Paysages et mémoire(s) de l'Antiquité : re-découverte, invention et oubli des paysages antiques $d u$ Biterrois, $X V I^{e}-X I X^{e}$ siècle. Directrice : Rita COMPATANGELO-SOUSSIGNAN.

\section{Université de Nantes}

\section{Thèse}

Ayala, Aurelio, Le North-West Mounted Police canadienne et ses auxiliaires métis, 1874-1900: une relation d'interdépendance? Directrice : Françoise LE JEUNE.

BERARDI, Riccardo, Féodalité laïque et seigneurie ecclésiastique dans l'Italie du Sud : de la Calabre des Normands à la guerre des Vêpres (1282). Directeurs : Annick PETERs-CUSTOT et Jean-Marie MARIN.
Bonamy, Alice, En quête d'une citoyenneté palestinienne: les élites économiques et politiques arabes à Jaffa et Haïfa dans la première moitié du XXe siècle. Directeurs : Bernard SALVAING et Henry LAURENS.

CHARRIER, Lény, L'influence du logis palatial du château des Ducs de Bretagne sur les hôtels intramuros de Nantes entre 1458 et 1532. Directeurs : Guy SAUPIN et Nicolas FAUCHERRE.

COUDREAU, Marin, Guerre et lutte contre les nuisibles en URSS, 1910-1940. Directeur : Michel CATALA.

HorN, Nelson, Étude des Livres XI et XII des Histoires philippiques de Trogue Pompée. Justin : La Composition historique autour de l'image d'Alexandre. Directeur : Bernard MinEO.

JENVRIN, Géraldine, L'interprétation de la notion de mécréance et le statut légal du non-musulman dans le commentaire coranique d'al-Qurtubî ( $m$. $671 / 1273)$ : traductions et commentaires. Directeur : John ToLAN.

KouAKOu, Bi Kakou Gildas Marc, La côte des Quaqua dans la traite négrière atlantique du XVIII siècle au XIXe siècle. Directeurs : Guy Saupin et Aka Kouamé.

LE, Van-Chien, Les frontières de l'Indochine : l'enjeu majeur des relations franco-siamoises (1858-1907). Directeur : Michel CATALA.

LEMER-FLEURY, Alice, L'Amérique du Nord britannique en métropole (17831815) : politiques coloniales et débats publics sur les colonies canadiennes en Angleterre et en Écosse. Directrice : Françoise LE JEUNE.

MaLANGin, Raphaël, Renoncer à l'Inde? Comptoirs, agents et aventuriers français aux Indes orientales à la fin du XVIII siècle. Directeur : Jacques WEBER.

MANSARE, Lamine, Histoire des Mandingues au Fouta Djallon du XVIII au XIX siècle : alliances et conflits? Acculturation et survie identitaire. Directeurs : Bernard SAlVAing et Seydou Magassouba.

NEveu, Elsa, Évolution des agricultures dans le Nord-Ouest de la France de l'âge du Bronze à l'époque romaine. 
Directeur : Serge CASSEN, co-encadrants : Cécile BRUn, Véronique ZECHMATTERNE.

Roux, Benoît, La France et la crise ivoirienne. Le processus des décisions françaises d'engagement militaire et de conclusion de l'Accord de Linas-Marcoussis (septembre 2002-février 2003). Directeur : Michel CATALA.

Sim, Gérald, La présence diplomatique et consulaire française aux États-Unis (1815-1904) : réseaux, acteurs, pratiques, regards. Directeur : Stanislas JEANNESSON.

\section{Université de Poitiers}

\section{Thèses}

BushreIDA EL-GAWHARI, Amal Le manuscrit de l'" Histoire chronologique du Royaume de Tripoli de Barbarie». Directeur: Thierry Sauzeau.

CAILLAUD, Emilie, Le fer: réseaux d'échange et circulation des matières premières métalliques en Aquitaine, du VIe siècle av. J.-C. au VIe siècle apr. J.-C. Directrice : Nadine Dieudonne-Glad; codirecteur : Didier BEZIAT (Toulouse).

Cousin, Michael, Correspondance et réseaux épistolaires latins de et en lien avec la péninsule ibérique (711-milieu $d u X^{e}$ siècle). Directeur: Thomas DESWARTE.

CRÉmIEU-AlCAN, Philippe, À l'ombre du roi : solidarité, résistance et mentalité à travers les délits forestiers en Guyenne au XVIII siècle. Directeur : Frédéric Chauvaud.

LAURENT, Sébastien, Troubadours et société en Aquitaine au XII siècle (1071-1199). Directeur : Martin AURELL; codirectrice Catalina GIRBEA (Bucarest).

PERSONNE, Marie-Laudes, L'émail contemporain de Limoges. Directrice : Claire BARBILLON.

Rigollet, Amélie, La famille de Briouze (XI'-XIII siècles). Directeur : Martin Aurell; codirecteur : Daniel PowER (Swansea).
SCHERER, Laurene, Images fantasmées, promotion et construction d'une nouvelle femme dans les années Trente. Directeur : Frédéric CHAUvaUd.

SURREAUX, Louis, La création contemporaine dans le Mobilier national et les Manufactures nationales-Gobelins, Beauvais, Savonnerie et Sèvres de 1945 à 2000. Directrice : Claire BARBILLON.

Vitolo, Anna Lisa, Le manuscrit illustré de Jaufre. Directeur : Martin AURELL; codirecteur : Giusi ZaNichelLI (Salerne).

\section{Université de Rennes 2}

\section{Habilitation à diriger des recherches}

ButTAY, Florence, Le pouvoir du lieu commun. Transmission et usages des savoirs et des images dans l'Europe de la Renaissance. Mémoire inédit : Peindre en leurs âmes des fantômes. Pédagogie et politique pendant les guerres de religion. Garant : Cédric MicHON.

LignereuX, Aurélien, L'empire de l'administration. Histoire sociale des gendarmes et des fonctionnaires expatriés en contexte impérial (XIX siècle). Avec un mémoire inédit intitulé : Les Impériaux, de l'Europe napoléonienne à la France post-impériale ( $1^{\text {re }}$ moitié du XIXe siècle). Garant: Pierre KARILACOHEN.

\section{Thèses}

Bellamy, Clément, La céramique indigène peinte de l'Incoronata. Étude typo-fonctionelle et anthropologie d'une production de l'âge du Fer en Italie méridionale. Directeur : Mario DENTI; co-directeur : Massimo OSANNA (Università di Foggia).

GAUTIER, Noémie, L'homme et l'animal domestique à Pompéi (II siècle av. J.-C.-79 apr. J.-C.) : essai d'histoire culturelle. Directeur : Christophe VENDRIES. 
LAUNAY, Vincent, Le roi en son duché : étude sur les relations entre le roi de France et l'aristocratie de Bretagne (1199-1328). Directeur : Florian MAZEL.

ReInBOLD, Aurélie, Dynamiques de la végétation et structuration des paysages. Étude interdisciplinaire des paysages agropastoraux des campagnes médiévales du nord de la Haute-Bretagne $\left(X I^{e}-X V I^{e}\right.$ siècle). Directeur : Florian MAZEL.

SILORET, Martin, La structuration partisane de l'écologie politique : une comparaison Bretagne-Pays de Galles (1974-1995). Directrice : Jacqueline SAINCLIVIER.

VILLETte, Mathilde, Physionomie d'un espace artisanal et processus de fabrication de la céramique à l'Âge du Fer sur la côte ionienne de l'Italie du Sud : l'atelier de potiers de l'Incoronata. Directeur : Mario DENTI.

\section{Université de Tours}

\section{Thèses}

REMY, Julie, L'organisation des territoires du quart nord-ouest de la Gaule (Bretagne et Pays de la Loire) à la fin de l'Âge du Fer (II -per siècle av. J.- C.). Directeur : Stéphan FICHTL.

NesRINe Si AmER, Aziza, Architecture des ksour de la vallée de Mzab : Essai d'histoire, de sémiotique et de médiation. Directeur : François-Olivier TOUATI.

Brochier, Diane, D'Azay-le-Rideau à Chenonceau: l'eau et la mise en scène de l'ensemble château-jardin à la Renaissance (1513-1560). Directeur : Alain SALAMAGNE. 